\title{
Evaluation of the antibacterial activity of tilmicosin-SLN against Streptococcus agalactiae: in vitro and in vivo studies
}

This article was published in the following Dove Press journal: International Journal of Nanomedicine

Luyan Zhu'

Xiaoxia Cao'

Qinxin $X u^{\prime}$

Jing $\mathrm{Su}^{\prime}$

Xihe $\mathrm{Li}^{2,3}$

Wenzhong Zhou'

'Department of Preventive Veterinary Medicine, College of Veterinary Medicine, China Agricultural University, Beijing, I00193, People's Republic of China; ${ }^{2}$ Research Center for Animal Genetic Resources of Mongolian Plateau School of Life Sciences, Inner Mongolia University, Hohhot, 01002I, People's Republic of China; ${ }^{3}$ Inner Mongolia Saikexing Institute of Breeding and Reproductive Biotechnology in Domestic Animals, Saikexing Breeding and Reproductive Biotechnology Co. Ltd., Hohhot, 0I I5 I7, People's Republic of China
Correspondence: Wenzhong Zhou Department of Preventive Veterinary Medicine, College of Veterinary Medicine, China Agricultural University, Beijing 100193, People's Republic of China Email zhouwz@cau.edu.cn
Background: Streptococcus and Staphylococcus are the major contagious organisms causing dairy cow mastitis. Our previous studies have demonstrated that solid lipid nanoparticles (SLNs) can effectively enhance the antimicrobial activity of tilmicosin against Staphylococcus. This study aimed to evaluate the antibacterial efficacy of tilmicosin-loaded SLN (Til-SLN) against Streptococcus agalactiae.

Methods: Til-SLN was prepared using a hot homogenization and ultrasonication method as described previously. Til-SLN was labeled with rhodamine B for nanoparticle tracking. In vitro antibacterial experiments were carried out by broth dilution technique. Pharmacokinetics of the drug and distribution of the nanoparticles in mammary gland were studied after subcutaneous injection in Kunming mice. The therapeutic study was conducted in a mouse mastitis model infected with $S$. agalactiae.

Results: The results showed that the diameter, polydispersity index, zeta potential, encapsulation efficiency, and loading capacity of the nanoparticles were not significantly affected by fluorescence labeling. Til-SLN showed a sustained and enhanced antibacterial activity in vitro. Til-SLN maintained a sustained drug concentration above $17 \mu \mathrm{g} / \mathrm{g}$ for at least 6 days in the mammary gland, as compared with only 3 days for the same amount of tilmicosin phosphate solution. The mean residence time and elimination half-life $\left(T_{1 / 2}\right)$ of Til-SLN were much longer than those of tilmicosin phosphate solution. Most of the nanoparticles remained at the injection site and a few were transferred to the mammary glands, indicating that the drug was slowly released at the injection site and then distributed to the mammary glands. SLN significantly enhanced the therapeutic efficacy of tilmicosin as determined by lower colony forming unit counts.

Conclusion: These results demonstrate that SLN could effectively enhance the antibacterial activity of tilmicosin against Streptococcus.

Keywords: tilmicosin, solid lipid nanoparticles, Streptococcus agalactiae, mastitis

\section{Introduction}

Mastitis is one of the most significant limiting factors to profitable dairying throughout the world. ${ }^{1}$ This pathology is typically caused by microbial intramammary infection that induces inflammation of the mammary gland. ${ }^{2}$ The inflammation reduces the quantity and quality of the milk produced by lactating cows. ${ }^{3}$

Mastitis in cows is mainly caused by bacteria. ${ }^{4,5}$ Mastitis-causing bacteria can be placed in two categories: the contagious organisms, including Staphylococcus aureus and Streptococcus agalactiae, and the environmental pathogens, including mainly coliforms and streptococci. ${ }^{4,6}$ Among these pathogens, Str. agalactiae can cause either acute, febrile disease or subacute, more chronic disease, both of which lead to 
diminishing milk production. ${ }^{6}$ This pathogen spreads widely within a herd during the milking process and is the chief cause of reinfection during the dry period. ${ }^{7}$

Antibiotic therapy remains the most widely used and effective method to control mastitis in dairy cows. ${ }^{6,8}$ Tilmicosin is a semi synthetic macrolide antibiotic and only used in the veterinary clinic. According to its bactericidal action spectrum and pharmacokinetic characteristics, tilmicosin is now widely used to combat mastitis in cattle and ovine. ${ }^{9}$ It has properties of broad antimicrobial spectrum including Gram-positive and Gram-negative bacteria associated with mastitis, ${ }^{10}$ low inhibitory concentration, and long elimination half time. ${ }^{11}$ At present, intramammary infusion of tilmicosin is implied for the treatment of mastitis. Subcutaneous (s.c.) administration is an alternate way for the treatment.

Studies showed that tilmicosin was effective against Sta. aureus mastitis, ${ }^{9,11}$ but was much less effective against Str. agalactiae mastitis. ${ }^{12}$ It was found that Str. agalactiae was less sensitive to tilmicosin than Sta. aureus. ${ }^{13}$ The minimal inhibitory concentration (MIC) of tilmicosin for Sta. aureus was $0.78 \mu \mathrm{g} / \mathrm{mL}$, whereas the MIC of tilmicosin for Str. agalactiae was $3.12 \mu \mathrm{g} / \mathrm{mL} .{ }^{13}$ Sensitivity of bacterium to antibiotics can predict the in vivo likely activity of the drug. Higher concentration of tilmicosin is required to eliminate Str. agalactiae in the mammary gland. Tilmicosin has poor penetration into areas of inflammation and microabscess formations ${ }^{14}$ and could be inactivated by binding with milk and serum components, ${ }^{14,15}$ both of which further reduce the concentration of drug available to destroy the bacteria. Inadequate therapeutic drug concentrations in the mammary gland could be one reason for treatment failure against Str. agalactiae mastitis. Another reason for treatment failure may be related to an insufficient residence time of the antibiotic in the mammary gland. The efficacy of an antibiotic, apart from the sensitivity of bacterium to the drug, is correlated with how long activity is maintained in the mammary gland and milk. ${ }^{5}$ All these characteristics suggest that the repeated use or a high dose of tilmicosin is more effective in the treatment of dairy cattle mastitis. Frequent administration is not only waste of time but also costly. Besides, frequent administration can cause stronger animal stress responses. A high dose can result in higher incidence of adverse effects such as acute cardiac toxicity. ${ }^{16}$ To address these issues, it is necessary to develop new strategies of delivery.

Solid lipid nanoparticles (SLNs), for their good biocompatibility, biodegradability and tolerability, ${ }^{17}$ are extensively explored in many medical areas, especially in drug delivery. Our previous studies demonstrated that the tilmicosin-loaded SLN (Til-SLN) prepared with hydrogenated castor oil (HCO) is a promising formulation to enhance the pharmacological activity and therapeutic efficacy of tilmicosin. Pharmacokinetics study after s.c. administration to mice demonstrated that SLN increased the bioavailability by 34-fold and extended the maintaining time of serum drug levels above 0.1 $\mu \mathrm{g} / \mathrm{mL}$ from 5 hours to 8 days. ${ }^{18}$ Moreover, therapeutic study in a mouse mastitis model showed that the SLN enhanced the antibacterial activity and therapeutic efficacy of tilmicosin against Sta. aureus with reduced dose and frequency of administration. ${ }^{19}$ The objective of the present study was to evaluate the antibacterial efficacy of Til-SLN against Str. agalactiae by in vitro and in vivo experiments.

\section{Materials and methods Materials}

Chemicals

$\mathrm{HCO}$ was purchased from Tongliao Tonghua Castor Chemical Co, Ltd (Inner Mongolia, China). Tilmicosin (93.3\%, w/w) was obtained from Jining Medicine Corp. (Jining, China). Polyvinyl alcohol (87\%-90\%, hydrolyzed, mol. wt. 30,000-70,000) was bought from Sigma-Aldrich (St Louis, MO, USA). Fetal bovine serum (FBS) was bought from Beijing Dingguo Tech. Co., Ltd (Beijing, China). The solvents used for high performance liquid chromatography (HPLC) were of liquid chromatography grade. Methanol and acetonitrile were purchased from Thermo Fisher Scientific (Waltham, MA, USA). The water for HPLC was prepared with a Milli-Q system (Millipore, Bedford, MA, USA). Tetrahydrofuran, methanol, and acetonitrile were bought from Sigma-Aldrich. Dibutylamine and phosphoric acid (85\%) were purchased from Beijing Chemical Reagent Co. (Beijing, China). Dibutylammonium phosphate solution was prepared by the addition of $168 \mathrm{~mL}$ of dibutylamine to $700 \mathrm{~mL}$ of phosphoric acid (85\%). The solution was allowed to cool and adjusted to $\mathrm{pH} 2.5 \mathrm{using}$ phosphoric acid (85\%), and the volume was made up to $1 \mathrm{~L}$ with water. Other chemicals and reagents not specified in the text were of analytical grade or equivalent.

\section{Animals and bacteria}

The experiments followed the guidelines of the Institutional Animal Care and Use Committee at China Agricultural University and were approved by the Committee. Kunming species mice (female, $30 \pm 1.2 \mathrm{~g}$ ) were purchased from the Medical Animal Test Center of Peking University and allowed to acclimatize for 1 week before use. They were kept at room temperature and at a relative humidity of $50 \%-60 \%$. Free access to food and water was allowed.

Str. agalactiae (C55913) was obtained from China Institute of Veterinary Drug Control (Beijing, China). Bacteria 
were streaked from glycerol-frozen stocks onto MuellerHinton agar (Biorad) plates supplemented with 5\% defibrinated sheep blood and incubated overnight at $35^{\circ} \mathrm{C}$. A single bacterial colony from the fresh plate was inoculated in the Mueller-Hinton broth (MHB) supplemented with 5\% FBS and grown at $35^{\circ} \mathrm{C}$ in a shaking incubator at $230 \mathrm{rpm}$ to an $\mathrm{OD}_{600}$ of 0.6 , which corresponded to $2.5 \times 10^{8}$ colony forming units $(\mathrm{CFU}) / \mathrm{mL}$, confirmed by plating serial dilutions. For the in vitro study, the bacteria were diluted in broth. For the in vivo study, the bacteria were collected by centrifugation at 4,000 rpm (Centrifuge $5810 \mathrm{R}$; Eppendorf, Hamburg, Germany) for 10 minutes at $4^{\circ} \mathrm{C}$ and resuspended in sterile PBS at different concentrations.

\section{Preparation and labeling of tilmicosin-SLN} Til-SLN was prepared by hot homogenization and ultrasonication method as described previously. ${ }^{18}$ Briefly, HCO $(0.8 \mathrm{~g})$ and tilmicosin $(0.2 \mathrm{~g})$ were heated in a boiling water bath. Preheated polyvinyl alcohol solution $(8 \mathrm{~mL}, 1 \%)$ was poured into the lipid phase under magnetic stirring for 2 minutes and sonicated for 2 minutes (VC X 750 VibraCell'm; Sonics \& Materials, Inc., Newtown, CT, USA) using the $3 \mathrm{~mm}$ microprobe with amplitude $35 \%$ to form a nanoemulsion, which was quickly poured into $100 \mathrm{~mL}$ cold water. The nanoparticles were collected by centrifugation at $11,603 \times g$ for 50 minutes at $4^{\circ} \mathrm{C}$ and washed three times with distilled water. The nanoparticles were suspended in $5 \mathrm{~mL}$ distilled water and lyophilized for 48 hours (LGJ-12 Freeze Dryer; Beijing Song Yuan Hua Xing Science Technology Development Co., Ltd, Beijing, China). The control SLN was formulated without tilmicosin.

In order to track SLN in mammary glands, SLN was labeled with rhodamine B. The labeled Til-SLN was prepared by the same method as mentioned above, except that $5 \mathrm{mg}$ of rhodamine B was added and dissolved in the melted lipid.

\section{Determination of particle size, polydispersity index (PDI) and zeta potential}

The particle size, PDI, and zeta potential analysis of SLN was performed by photon correlation spectroscopy using Zetasizer Nano ZS90 (Malvern Instruments, Malvern, UK). The samples were suspended in distilled water at a concentration of $2.67 \mathrm{mg} / \mathrm{mL}$ for particle size and PDI determination and $0.30 \mathrm{mg} / \mathrm{mL}$ for zeta potential determination.

\section{Microscopic studies}

For optical microscopic analysis, $1 \mathrm{mg}$ of SLN was suspended in $1 \mathrm{~mL}$ of double distilled water. One microliter of the suspension was placed on a microscope slide, and the particles were dried at room temperature. Photomicrographs of the nanoparticles were taken using an inverted optical microscope (Olympus 1×71; Olympus Corporation, Tokyo, Japan). The fluorescence emission spectrum of the labeled Til-SLN was obtained using an LS 55 luminescence spectrometer (PerkinElmer Inc., Shelton, CT, USA).

\section{Determination of loading capacity (LC) and encapsulation efficiency (EE)}

To determine the drug content in the nanoparticles, a weighed amount of freeze-dried SLN was dissolved in chloroform, and the solution was analyzed directly at $292 \mathrm{~nm}$ using ultraviolet spectrophotometer (U-1800; Hitachi Tech Co., Tokyo, Japan). The control nanoparticles formulated without tilmicosin were treated similarly as blanks for measurements. The assay was repeated three times using different samples from independent preparations. Drug LC and EE are defined as follows:

$$
\begin{aligned}
& \mathrm{LC}=\frac{\text { Weight of tilmicosin in SLN }}{\text { Weight of SLN }} \times 100 \% . \\
& \mathrm{EE}=\frac{\text { Weight of tilmicosin in SLN }}{\text { Weight of tilmicosin added }} \times 100 \% .
\end{aligned}
$$

\section{Antibacterial activity studies}

The MIC and minimum bactericidal concentration (MBC) were determined by microdilution. MHB supplemented with $5 \%$ FBS was used as a test medium. Briefly, $20 \mu \mathrm{L}$ Str. agalactiae containing $0.5 \mathrm{McF}$ arland $\left(1.5 \times 10^{8} \mathrm{CFU} / \mathrm{mL}\right)$ of organisms in test medium was added to $2 \mathrm{~mL}$ test medium in $4 \mathrm{~mL}$ tubes containing native tilmicosin or Til-SLN with various drug concentrations ranging from 0.2 to $20 \mu \mathrm{g} / \mathrm{mL}$. Tubes were incubated at $35^{\circ} \mathrm{C}$ for 12 hours under aerobic conditions with shaking at $130 \mathrm{rpm}$. The MIC of each group was defined as the lowest concentration inhibiting visible growth of bacteria after overnight incubation of the cultures at $35^{\circ} \mathrm{C}$. The $\mathrm{MBC}$ was measured by subculturing the broths used for MIC determination onto fresh agar plates supplemented with 5\% defibrinated sheep blood. The MBC is the lowest concentration of the drug that results in killing $99.9 \%$ of the bacteria being tested. All the experiments were conducted in triplicate.

Sustained antibacterial studies were conducted by broth dilution technique. Twenty microliters of bacterial culture containing $0.5 \mathrm{McF}$ arland $\left(1.5 \times 10^{8} \mathrm{CFU} / \mathrm{mL}\right)$ of organisms was added to $2 \mathrm{~mL}$ MHB medium supplemented with $5 \%$ 
FBS in a $4 \mathrm{~mL}$ tube containing tilmicosin solution or Til-SLN with the same drug concentration $(0.9 \mu \mathrm{g} / \mathrm{mL})$. Blank nanoparticles $(8.2 \mu \mathrm{g} / \mathrm{mL})$, blank nanoparticles $(8.2 \mu \mathrm{g} / \mathrm{mL})$ with tilmicosin $(0.9 \mu \mathrm{g} / \mathrm{mL})$, and $\mathrm{MHB}(5 \% \mathrm{FBS})$ served as controls. The mixtures were incubated at $35^{\circ} \mathrm{C}$ in an incubator with shaking (130 rpm). At fixed time points (12, 24, 36 , 48, and 60 hours), serial ten time dilutions were made, and spread plating was performed on Mueller-Hinton agar plates supplemented with 5\% defibrinated sheep blood and incubated at $35^{\circ} \mathrm{C}$. After 24 hours, the colonies were counted by naked eyes. All the experiments were conducted in triplicate. Growth graphs were plotted by taking the number of colonies vs time for comparing the action of Til-SLN with native tilmicosin on the growth of Str. agalactiae.

\section{Pharmacokinetics of tilmicosin in mammary glands}

Before initiation of the experiment, lactating mice were randomly divided into 16 groups with five animals in each group. Eight groups were used to determine the pharmacokinetic parameters of Til-SLN and the others for native tilmicosin. Different groups of mice were injected with one dose of tilmicosin ( $60 \mathrm{mg} / \mathrm{kg}$ of body weight) in the form of either Til-SLN suspended in $0.2 \mathrm{~mL} 0.9 \%(\mathrm{w} / \mathrm{v}) \mathrm{NaCl}$ solution or native tilmicosin dissolved in the same solution, into the dorsal s.c. tissues. At different time points $(0.5,1,1.5,2,3,4,5$, and 6 days $)$, mammary glands were harvested and stored at $-80^{\circ} \mathrm{C}$.

Mammary glands were minced and homogenized in $1 \mathrm{~mL}$ ultra pure water. Three hundred microliters of homogenate was accurately mixed with the same amount of acetonitrile and shaken for 30 minutes (250 rpm). Centrifugation was performed for 5 minutes at $1,844 \times g$. The supernatant was transferred into a $1.5 \mathrm{~mL}$ tube. The tissue pellet was resuspended in $300 \mu \mathrm{L}$ of monobasic potassium phosphate buffer plus $300 \mu \mathrm{L}$ of acetonitrile, shaken for 30 minutes (250 rpm) and centrifuged as above. Supernatants were combined and centrifuged again at $14,462 \times g$ for 10 minutes to remove debris. The processed sample solution was filtered with $0.22 \mu \mathrm{m}$ syringe filters, and $400 \mu \mathrm{L}$ was used for HPLC analysis.

Concentrations of tilmicosin in mammary glands were measured by HPLC with ultraviolet spectroscopy using Agilent HPLC series 1,100 with UV detector (DAD), equipped with the ZORBAX Eclipse XDB C18 column (4.6-250 mm, $5 \mathrm{~mL}$ ). Chromatographic conditions were as follows: the mobile phase was prepared by adding $135 \mathrm{~mL}$ acetonitrile, $55 \mathrm{~mL}$ tetrahydrofuran, and $25 \mathrm{~mL}$ of $1 \mathrm{M}$ dibutylammonium phosphate to ultra-pure water to a final volume of $1,000 \mathrm{~mL}$; the flow rate was $1.0 \mathrm{~mL} / \mathrm{min}$, the injection volume was $50 \mu \mathrm{L}$, and the detection wavelength was $287 \mathrm{~nm}$.

\section{Intramammary distribution of SLN}

Six lactating mice were randomly divided into two groups. One group was injected s.c. with rhodamine B-labeled Til-SLN ( $84.3 \mathrm{mg} / \mathrm{kg}$, which is equivalent to $10 \mathrm{mg} / \mathrm{kg}$ of pure tilmicosin). Another group was treated as control with rhodamine B solution $(0.2 \mathrm{mg} / \mathrm{kg})$. Tissues were harvested from the mammary glands and the injection sites at 3 days after injection. The tissues were cut into very small pieces with scissors and put onto a slide and squeezed with a coverslip. Photomicrographs were taken using an inverted optical microscope (Olympus $1 \times 71$; Olympus Corporation) with the fluorescence emission spectrum obtained using an LS 55 luminescence spectrometer (PerkinElmer Inc.).

\section{Mouse mastitis model}

Mice were infected with Str. agalactiae by intramammary injection as described previously. ${ }^{19}$ Briefly, mice (16-18 days after parturition) were removed from their pups 1-2 hours before bacterial inoculation. A $1 \mathrm{~mL}$ syringe with a 33-gage blunt needle was used for inoculation. After ether anesthesia, the teat of L4 (on the left) and R4 (on the right) was disinfected with $75 \%$ ethanol, and then, $1 \times 10^{5}$, $1 \times 10^{6}, 1 \times 10^{7}$, and $1 \times 10^{8} \mathrm{CFU}$ of Str. agalactiae suspended in $100 \mu \mathrm{L}$ sterile PBS or $100 \mu \mathrm{L}$ plain sterile PBS was infused into each mammary duct. The mice were euthanized 24 hours postinfection for bacterium quantitation. Mammary glands were removed aseptically, weighed, and homogenized in PBS to a final volume of $1.5 \mathrm{~mL}$. The bacterial counts $(\mathrm{CFU})$ were determined by plating logarithmic dilutions of the tissue samples $(100 \mu \mathrm{L})$ on Mueller-Hinton agar plates supplemented with $5 \%$ defibrinated sheep blood with detection limits of $900 \mathrm{CFU} / \mathrm{g}$. A desirable experimental condition for subsequent therapeutic efficacy studies was selected.

\section{Therapeutic treatment schedules}

Sixty lactating mice were randomly divided into ten groups and treated as follows: group NC (non-infected control group and treated with $0.9 \%[\mathrm{w} / \mathrm{v}] \mathrm{NaCl}$ solution); group IC (infected control group and treated with $0.9 \%[\mathrm{w} / \mathrm{v}] \mathrm{NaCl}$ solution); group Native1 (infected group and treated with native tilmicosin at a dose of $20 \mathrm{mg} / \mathrm{kg}$ ); group Native 2 (infected group and treated with native tilmicosin at a dose of $10 \mathrm{mg} / \mathrm{kg}$ ); group SLN1 (infected group and treated with Til-SLN at a nanoparticle dose of $168.6 \mathrm{mg} / \mathrm{kg}$, which is equivalent to $20 \mathrm{mg} / \mathrm{kg}$ of pure tilmicosin); and group SLN2 (infected group and treated with Til-SLN at a nanoparticle dose of $84.3 \mathrm{mg} / \mathrm{kg}$, which is equivalent to $10 \mathrm{mg} / \mathrm{kg}$ of pure tilmicosin). Therapeutic treatment was done 1 day 
postinfection. The mice were euthanized at 3 or 6 day post-therapy. Mammary glands were aseptically collected, weighed, and homogenized in PBS to a final volume of $1.5 \mathrm{~mL}$. The bacterial counts (CFU) were determined by plating $100 \mu \mathrm{L}$ of samples on Mueller-Hinton agar plates supplemented with 5\% defibrinated sheep blood with detection limits of 1,100 and 1,500 CFU/g, since the weight of mammary glands changed. If the CFU was too high to count, further serial logarithmic dilutions were made. CFU counts were transformed as base 10 logarithmic values. Graphs were plotted by taking the number of colonies vs different time points for comparing the action of Til-SLN with native tilmicosin on the mastitis therapy.

\section{Statistical methods}

Results were analyzed and expressed as mean \pm SD. Statistical analysis was performed using the Student's $t$-test. The differences were considered significant at $P<0.05$.

The values of maximum serum concentration $\left(\mathrm{C}_{\max }\right)$ and time to peak serum concentration $\left(\mathrm{T}_{\max }\right)$ were obtained directly from the concentration-time plotting. The area under the concentration-time curve $\left(\mathrm{AUC}_{0 \text {-inf }}\right)$, mean residence time (MRT), and elimination half-life $\left(\mathrm{T}_{1 / 2}\right)$ were analyzed based on noncompartmental pharmacokinetics statistical moment. The relative bioavailability at infinity was calculated as: Fr $=\mathrm{AUC}_{\mathrm{NFX} \text {-SLn, 0-inf }} / \mathrm{AUC}_{\mathrm{NFX}, 0 \text {-inf* }}$ Microsoft Excel (2003) (Microsoft Corporation, Redmond, WA, USA) was used for all statistical analyses.

\section{Results}

\section{Physicochemical characteristics of SLN}

Photomicrographs showed that both labeled and unlabeled Til-SLNs were well dispersed (Figure 1). Fluorescence microscopy image showed that Til-SLN was clearly labeled with rhodamine B. Photon correlation spectroscopy analysis indicated that the values of mean diameter, PDI, zeta potential, LC, and EE were not significantly affected by rhodamine B (Table 1).

\section{In vitro antibacterial activity}

In vitro antibacterial activity study showed that both the MIC and MBC values of Til-SLN were higher than those of the native tilmicosin at 12 hours (Table 2). Sustained antibacterial activity is depicted in Figure 2. The figure gives a comparison of the overall trend of antibacterial activity from 12 to 48 hours. At early time points (12 and 24 hours), Til-SLN was less effective, but at the later time points (36 and 48 hours), Til-SLN was much more effective than native tilmicosin solution. Colony number of native tilmicosin solution sharply increased from 24 to 36 hours and almost reached the levels of control. The two curves representing the control and blank SLN were in the same trend. Native tilmicosin and the mixture of native tilmicosin with blank SLN did not have significant difference in the colony numbers at all time points.

\section{The pharmacokinetics of tilmicosin in mammary glands}

Figure 3 shows the concentration of tilmicosin vs time plotting following s.c. injection of Til-SLN and native tilmicosin. Maximum levels of drug concentrations were attained at 2 days after injection in both native tilmicosin group and Til-SLN group. After that, the drug concentrations in native tilmicosin group steadily declined. In contrast, the Til-SLN group showed a sustained drug concentration above $17 \mu \mathrm{g} / \mathrm{g}$ for at least 6 days. Pharmacokinetic analysis indicated that the elimination half-life $\left(\mathrm{T}_{1 / 2}\right), \mathrm{MRT}$, and $\mathrm{AUC}_{0 \text {-inf }}$ of the Til-SLN group increased significantly compared to those of native tilmicosin. SLN enhanced the relative bioavailability of tilmicosin by 6-fold as reflected by $\mathrm{AUC}_{0-\text { inf }}$. The maximum serum concentrations $\left(\mathrm{C}_{\max }\right)$ of the two groups showed no significant difference (Table 3 ).
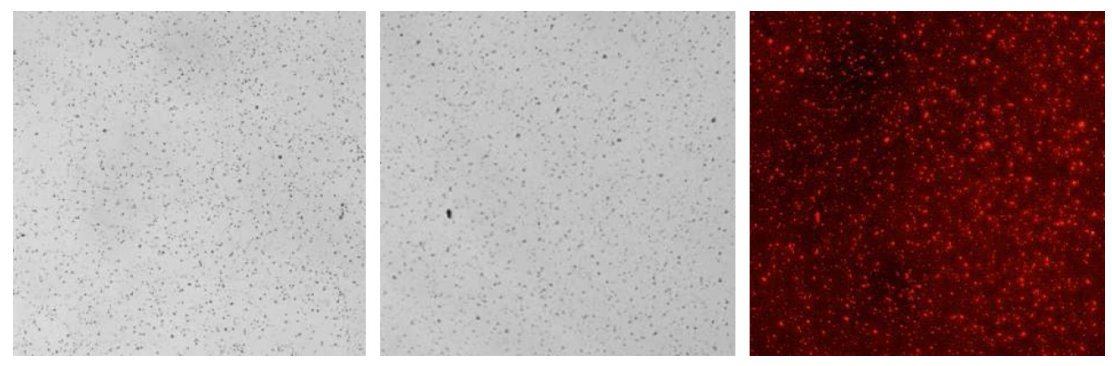

Figure I Photographs of tilmicosin-SLN and labeled tilmicosin-SLN (magnification was 10×40).

Notes: Left, optical microscopy image of tilmicosin-SLN; middle, optical microscopy image of labeled tilmicosin-SLN; and right, fluorescence microscopy image of labeled tilmicosin-SLN. The fluorescence microscopy image of labeled tilmicosin-SLN was obtained at the same area of normal light.

Abbreviation: SLN, solid lipid nanoparticle. 
Table I Characteristics of SLNs (mean \pm SD, $n=3$ )

\begin{tabular}{llllll}
\hline & EE (\%) & LC (\%) & MD (nm) & PDI & ZP (mV) \\
\hline Til-SLN & $59.4 \pm 2.4$ & $11.2 \pm 0.30$ & $343 \pm 26$ & $0.41 \pm 0.05$ & $-7.9 \pm 0.4$ \\
RB-Til-SLN & $57.4 \pm 3.8$ & $10.8 \pm 0.24$ & $365 \pm 49$ & $0.45 \pm 0.18$ & $-8.5 \pm 0.5$ \\
\hline
\end{tabular}

Abbreviations: EE, encapsulation efficiency; LC, loading capacity; MD, mean diameter; PDI, polydispersity index; RB-Til-SLN, rhodamine B labeled tilmicosin-SLN; SLN, solid lipid nanoparticle; Til-SLN, tilmicosin-SLN; ZP, zeta potential.

\section{Intramammary distribution of SLN}

Fluorescent-labeled Til-SLN were observed in the mammary gland after s.c. injection of the nanoparticles (Figure 4, left), while the majority of the nanoparticles were found to remain at the injection site (Figure 4, middle). Injection of rhodamine B solution resulted in moderate red fluorescence in mammary glands (Figure 4, right).

\section{Levels of infection}

Table 4 shows the CFU numbers of the mammary glands of the four inoculations 1 day after infection. The inoculations of $10^{5}$ and $10^{6}$ were so insufficient that the variations in the number of bacterial colonization are big and in some mammary gland, they were under the test line $(900 \mathrm{CFU} / \mathrm{g})$. The inoculation of $10^{7}$ was adequate, and the infection could be sustained for at least 6 days (Figure 5). When the inoculation increased to $10^{8}$, the CFU numbers had no significant change, but the damage for the mammary glands was so serious that pustules formed. Therefore, $1 \times 10^{7} \mathrm{CFU} / \mathrm{gland}$ was selected as the experimental condition for subsequent therapeutic efficacy studies. No animals died during the experiment and no bacterial colonization was observed in the control mice.

\section{The therapeutic efficacy of Til-SLN}

As shown in Figure 6, all the SLN groups had lower level of CFU than the native tilmicosin groups $(P<0.05)$. At 3 days, all the treatment groups showed a significant effect compared with group IC $(P<0.05)$. At 6 days, the SLN1 $(20 \mathrm{mg} / \mathrm{kg})$ group had a CFU level under detection limit $(1,500 \mathrm{CFU} / \mathrm{g})$. The difference in CFU levels between

Table 2 MIC and MBC of native tilmicosin and tilmicosin-SLN

\begin{tabular}{lll}
\hline & MIC $(\mu \mathrm{g} / \mathrm{mL})$ & MBC $(\mu \mathrm{g} / \mathrm{mL})$ \\
\hline Native tilmicosin & 0.9 & 8 \\
Tilmicosin-SLN & 1.2 & 12.3 \\
\hline
\end{tabular}

Abbreviations: $\mathrm{MBC}$, minimum bactericidal concentration; MIC, minimal inhibitory concentration; SLN, solid lipid nanoparticle.

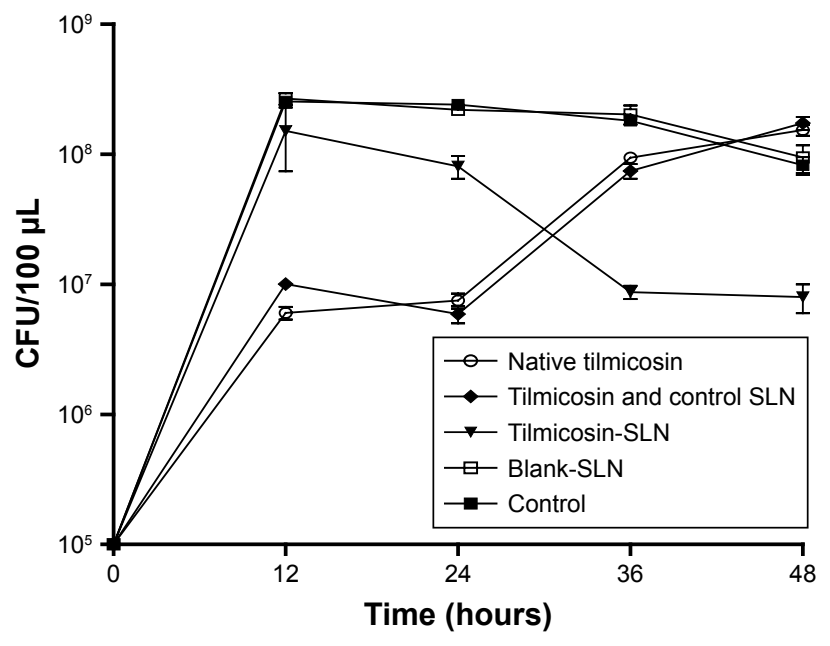

Figure 2 Sustained antibacterial activity of native tilmicosin and tilmicosin-SLN (drug concentration: $0.9 \mu \mathrm{g} / \mathrm{mL}$ ).

Abbreviations: CFU, colony forming unit; SLN, solid lipid nanoparticle.

Native1 $(20 \mathrm{mg} / \mathrm{kg})$ group and SLN2 $(10 \mathrm{mg} / \mathrm{kg})$ group was statistically insignificant. The number of Str. agalactiae in the mammary glands of Native $2(10 \mathrm{mg} / \mathrm{kg})$ group reached the same level as in the IC ( $\mathrm{NaCl}$ solution) group. Control SLN group and $\mathrm{IC}(\mathrm{NaCl}$ solution) group did not have significant difference in the colony numbers at 3 and 6 days.

\section{Discussion}

In this study, Til-SLN and labeled Til-SLN were prepared by hot homogenization and ultrasonication method. The physicochemical characteristics of the labeled Til-SLN was very similar to unlabeled tilmicosin-SLN, suggesting that labeled Til-SLN can be used to track SLN in vivo.

Higher MIC and MBC values of Til-SLN than native tilmicosin at 12 hours could be due to the reason that the drug encapsulated in nanoparticles was not released completely.

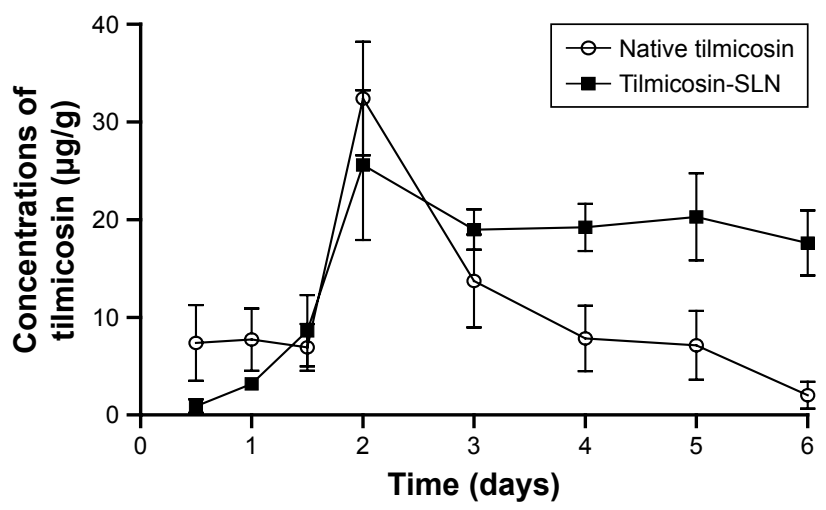

Figure 3 Concentrations of tilmicosin in mammary glands after s.c. injection $(60 \mathrm{mg} / \mathrm{kg})$.

Abbreviations: s.c., subcutaneous; SLN, solid lipid nanoparticle. 
Table 3 Pharmacokinetic after s.c. injection in mice (mean $\pm S D$, $\mathrm{n}=5$ )

\begin{tabular}{lll}
\hline $\begin{array}{l}\text { Pharmacokinetic } \\
\text { value }\end{array}$ & Tilmicosin-SLN & $\begin{array}{l}\text { Native } \\
\text { tilmicosin }\end{array}$ \\
\hline AUC $_{0 \text {-inf }}$ & $1 \mathrm{I}, 815.91 \pm 5,652.70^{\mathrm{a}}$ & $\mathrm{I}, 969.40 \pm 2 \mathrm{I} 6.54$ \\
$\mathrm{MRT}$ (hours) & $425.33 \pm 209.25^{\mathrm{a}}$ & $100.73 \pm 23.3 \mathrm{I}$ \\
$\mathrm{C}_{\max }$ ( $\left.\mu \mathrm{g} / \mathrm{g}\right)$ & $25.57 \pm 7.65^{\mathrm{b}}$ & $32.37 \pm 5.8 \mathrm{I}$ \\
$\mathrm{T}_{\max }$ (hours) & $48^{\mathrm{b}}$ & 48 \\
$\mathrm{~T}_{1 / 2}$ (hours) & $363.28 \pm 170.26^{\mathrm{a}}$ & $56.39 \pm 23.02$ \\
$\mathrm{Fr}$ & $6.00^{\mathrm{a}}$ & $\mathrm{I}$ \\
\hline
\end{tabular}

Notes: ${ }^{2}$ Statistical significances compared with native tilmicosin are $P<0.05$. bStatistical significances compared with native tilmicosin are $P>0.05$.

Abbreviations: $A \cup C_{0 \text {-inf }}$ the area under the concentration-time curve; $C_{\max }$, the values of maximum serum concentration; $\mathrm{Fr}$, the relative bioavailability at infinity: MRT, mean residence time; s.c., subcutaneous; SLN, solid lipid nanoparticle; $T_{1 / 2}$, elimination half-life; $\mathrm{T}_{\max }$, time to peak mammary glands concentration.

This result is similar to that obtained in our previous work. ${ }^{19}$ Til-SLN had a certain burst release within 2 hours and then began to release tilmicosin slowly. This initial burst could be caused by the adsorption of tilmicosin on the surface of nanoparticles and part of the drug leaching from the nanoparticle. ${ }^{20}$ In the actual treatment process, fast release is conducive to achieve effective plasma concentration in a short time.

Sustained-release antibacterial studies showed that at 12 and 24 hours, Til-SLN was less effective. Lower drug concentration might account for the less effectiveness of Til-SLN at early time points because only a part of the drug is released from the nanoparticles. However, sustained drug concentration is required to control the growth of bacteria for a longer period of time. After 12 hours, owing to its sustained release, Til-SLN was advantageous over native tilmicosin. Native tilmicosin became less effective after 24 hours. This result is similar to the result of the antibacterial capacity of Til-SLN against Str. agalactiae compared with native tilmicosin. The control group and the blank SLN group had the same growth kinetics of Str. agalactiae, while the native tilmicosin group had the same growth kinetics of Str. Agalactiae as that of the mixture of native tilmicosin and blank SLN group. This further confirmed that Til-SLN remained effective for a longer period of time mainly owing to the sustained release of the drug.

Pharmacokinetic study after s.c. administration demonstrated that SLN significantly extended the MRT, increased the bioavailability, and prolonged the maximum $T_{1 / 2}$ of tilmicosin in mammary glands. AUC reflects the degree of drug absorption. SLN significantly improved the body's absorption of tilmicosin. Moreover, the MRT, the maximum $\mathrm{T}_{1 / 2}$, and the bioavailability of tilmicosin in mammary glands were longer or higher compared with those in serum. ${ }^{18}$ Tilmicosin has special pharmacokinetic characteristics to accumulate in the breast and milk from blood to maintain a long residence time. ${ }^{13}$ Since the bactericidal activity of tilmicosin is time dependent, a longer drug action time is essential for the treatment of infection in mammary glands. The SLN significantly prolonged the action time of the drug in mammary glands, which suggests that the SLN could enhance the therapeutic efficacy and also reduce the frequency of drug administration.

Through tracking the labeled tilmicosin-SLN, we found that the majority of the SLN remained at the injection sites, suggesting that most of the drug was released from the nanoparticles locally at the injection site. The ability of SLN to enhance the therapeutic efficacy of tilmicosin should be mainly achieved through sustained releasing of the drug. A small part of the nanoparticles was transferred to the mammary glands. These drug-loaded nanoparticles in mammary glands could play a minor role in the antibacterial activity. ${ }^{21}$

It was reported that s.c. administration of tilmicosin was not very effective for dry cows against Str. Agalactiae. ${ }^{11}$ In our study, the bacterial content in the mammary glands of all treatment groups was maintained at high levels (above $10^{5} \mathrm{CFU} / \mathrm{g}$ ), except in the SLN1 group. The SLN1 group showed a significantly lower bacterial content in all
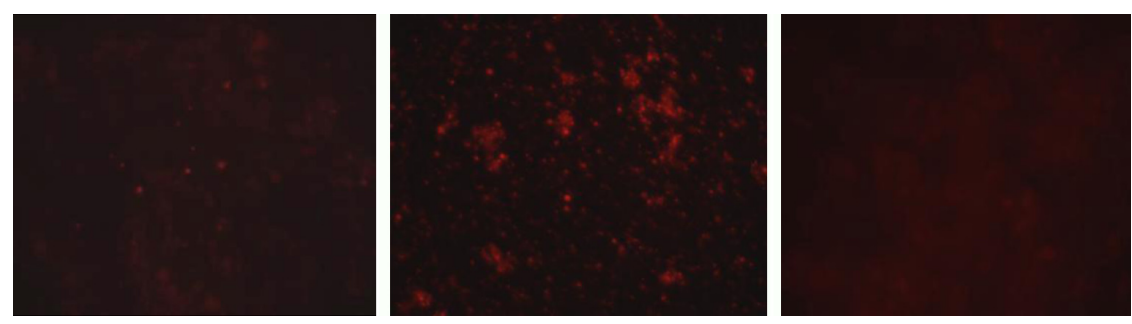

Figure 4 Fluorescence microscopy images of mammary gland slices.

Notes: Left, treated with labeled tilmicosin-SLN; middle, injection site; and right, treated with rhodamine B solution.

Abbreviation: SLN, solid lipid nanoparticle. 
Table 4 The number of Streptococcus agalactiae in mammary glands (CFU/g) after they are infected with different inoculation quantities at $\mathrm{I}$ day (mean $\pm \mathrm{SD}, \mathrm{n}=4$ )

\begin{tabular}{ll}
\hline Inoculums $(\mathrm{CFU} / 100 \mu \mathrm{L})$ & CFU/g \\
\hline $10^{5}$ & $(1.22 \pm 0.88) \times 10^{3}$ \\
$10^{6}$ & $(1.52 \pm 0.98) \times 10^{4}$ \\
$10^{7}$ & $(8.53 \pm 2.76) \times 10^{7}$ \\
$10^{8}$ & $(7.48 \pm 3.83) \times 10^{7}$ \\
\hline
\end{tabular}

Abbreviation: CFU, colony forming unit.

mammary glands, which was under the detection limit. The efficacy of the therapy appears to be more influenced by pharmacokinetics. The pharmacokinetics of native tilmicosin and Til-SLN explain the improvement of treatment effect of Til-SLN. This enhanced efficacy was related to the sustained release effect of the nanoparticles in vivo. Nanoparticles gradually release the encapsulated drugs, prolong the action time, and enhance the role of drug components, so as to achieve the desired treatment effect. ${ }^{19}$ Group IC and blank SLN did not have significant difference in the colony numbers at 3 and 6 days, indicating that the nanoparticles themselves did not have bactericidal effect.

\section{Conclusion}

SLN effectively enhanced the therapeutic efficacy of tilmicosin against Str. agalactiae mastitis. Sustained release of the drug played a major role, while the drug-loaded nanoparticles transported to mammary glands could play a minor role in the antibacterial activity. Til-SLN would be a promising

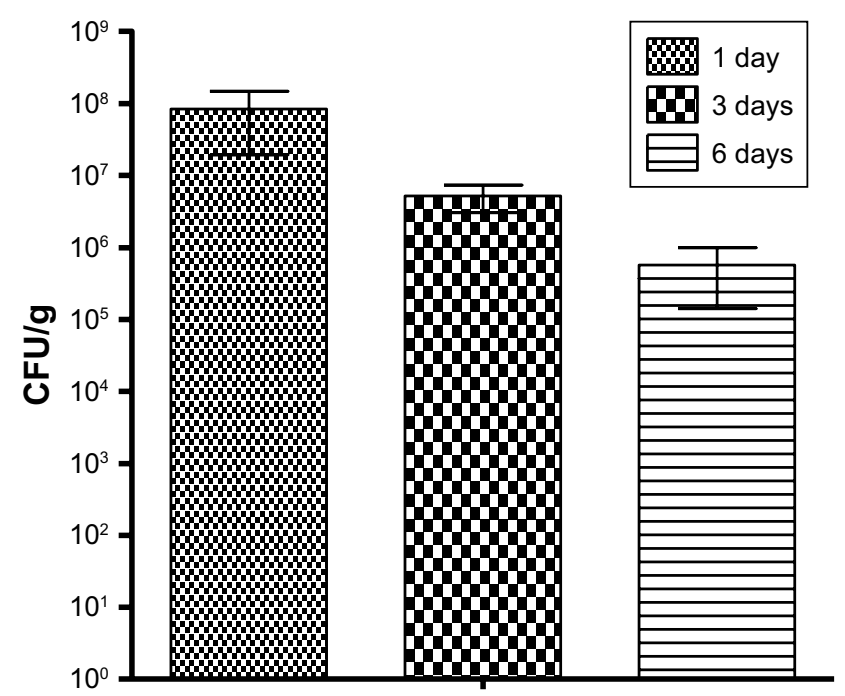

Figure 5 The number of Streptococcus agalactiae in mammary glands that were infected with $\mathrm{I} \times 10^{7} \mathrm{CFU} / \mathrm{I} 00 \mu \mathrm{L}$ per mammary gland at $\mathrm{I}, 3$, and 6 days (mean $\pm \mathrm{SD}$, $\mathrm{n}=5$ ).

Abbreviation: CFU, colony forming unit.
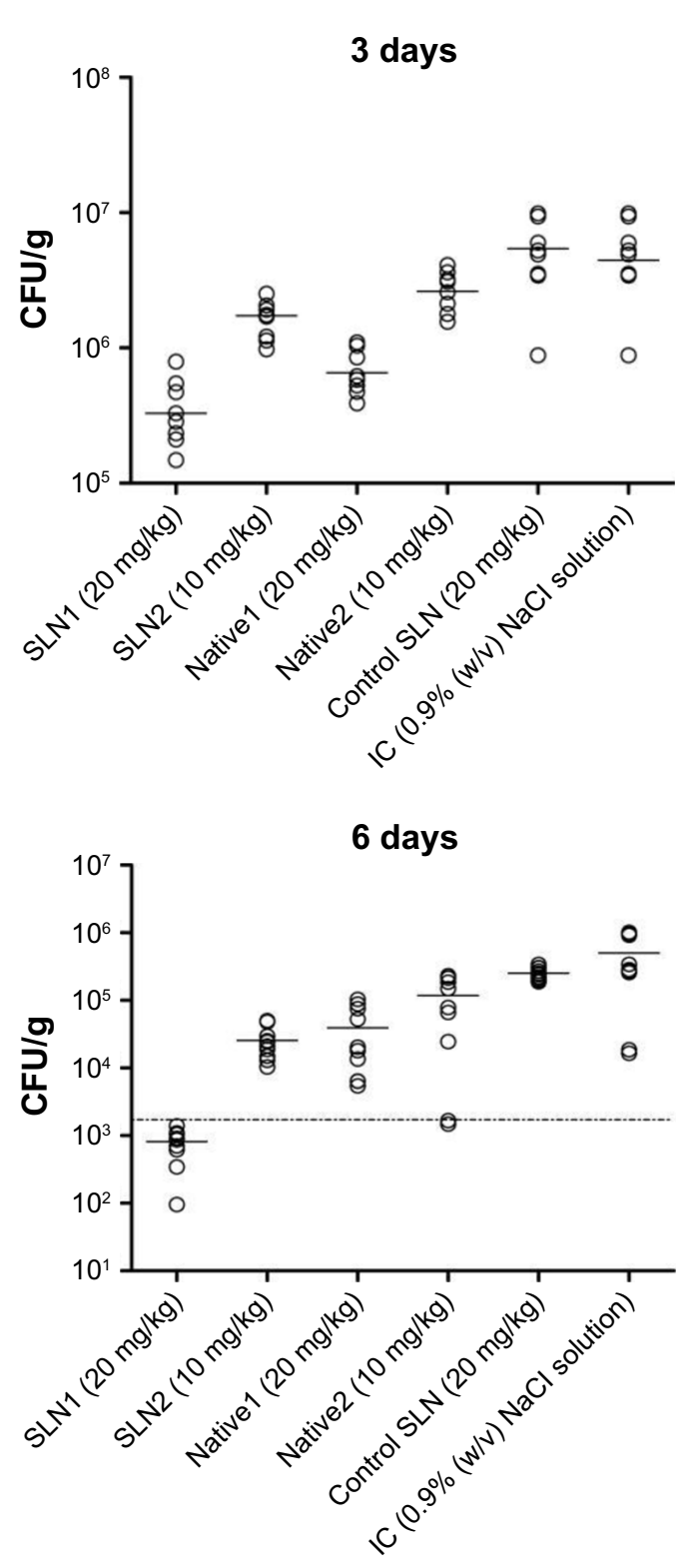

Figure 6 CFU counts in mammary glands after treatment.

Note: The horizontal line indicates the CFU detection limit.

Abbreviations: $\mathrm{CFU}$, colony forming unit; IC, infected control group and treated with $0.9 \%[\mathrm{w} / \mathrm{v}] \mathrm{NaCl}$ solution; SLN, solid lipid nanoparticle.

formulation for prophylaxis and therapy of Str. agalactiae causing cow mastitis.

\section{Disclosure}

The authors report no conflicts of interest in this work.

\section{References}

1. Calvinho LF, Almeida RA, Oliver SP. Potential virulence factors of Streptococcus dysgalactiae associated with bovine mastitis. Vet Microbiol. 1998;61(1-2):93-110.

2. Brouillette E, Malouin F. The pathogenesis and control of Staphylococcus aureus-induced mastitis: study models in the mouse. Microbes Infect. 2005;7(3):560-568. 
3. Brouillette E, Grondin G, Talbot BG, Malouin F. Inflammatory cell infiltration as an indicator of Staphylococcus aureus infection and therapeutic efficacy in experimental mouse mastitis. Vet Immunol Immunopathol. 2005;104(3):163-169.

4. Notebaert S, Meyer E. Mouse models to study the pathogenesis and control of bovine mastitis. A review. Vet Q. 2006;28(1):2-13.

5. Gruet P, Maincent P, Berthelot X, Kaltsatos V. Bovine mastitis and intramammary drug delivery: review and perspectives. Adv Drug Deliv Rev. 2001;50(3):245-259.

6. Guérin-Faublée V, Tardy F, Bouveron C, Carret G. Antimicrobial susceptibility of Streptococcus species isolated from clinical mastitis in dairy cows. Int J Antimicrob Agents. 2002;19(3):219-226.

7. Trigo G, Dinis M, França A, et al. Leukocyte populations and cytokine expression in the mammary gland in a mouse model of Streptococcus agalactiae mastitis. J Med Microbiol. 2009;58(Pt 7):951-958.

8. Eberhart RJ. Management of dry cows to reduce mastitis. J Dairy Sci. 1986;69(6):1721-1732.

9. Naccari F, Martino D, Giofrè F, Passantino A, de Montis P. Therapeutic efficacy of tilmicosin in ovine mammary infections. Small Rumin Res. 2003;47(1):1-9.

10. Modric S, Webb AI, Davidson M. Effect of respiratory tract disease on pharmacokinetics of tilmicosin in rats. Lab Anim Sci. 1999;49(3): 248-253.

11. Nickerson SC, Owens WE, Fox LK, et al. Comparison of tilmicosin and cephapirin as therapeutics for Staphylococcus aureus mastitis at dry-off. J Dairy Sci. 1999;82(4):696-703.

12. Mohammadsadegh M. Impact of intramammary tilmicosin infusion as a dry cow therapy. $J$ Vet Pharmacol Ther. 2018;41(1):22-27.
13. Ziv G, Shem-Tov M, Glickman A, Winkler M, Saran A. Tilmicosin antibacterial activity and pharmacokinetics in cows. J Vet Pharmacol Ther. 1995;18(5):340-345.

14. Ehinger AM, Kietzmann M. Pharmacokinetic aspects of mastitis therapy. Berl Munch Tierarztl Wochenschr. 1998;111(9):337-343.

15. Owens WE, Watts JL. Effects of milk on activity of antimicrobics against Staphylococcus aureus isolated from bovine udders. J Dairy Sci. 1987;70(9):1946-1951.

16. Ramadan A. Pharmacokinetics of tilmicosin in serum and milk of goats. Res Vet Sci. 1997;62(1):48-50.

17. Xie S, Wang S, Zhu L, Wang F, Zhou W. The effect of glycolic acid monomer ratio on the emulsifying activity of PLGA in preparation of protein-loaded SLN. Colloids Surf B Biointerfaces. 2009;74(1): 358-361.

18. Han C, Qi CM, Zhao BK, et al. Hydrogenated castor oil nanoparticles as carriers for the subcutaneous administration of tilmicosin: in vitro and in vivo studies. J Vet Pharmacol Ther. 2009;32(2):116-123.

19. Wang XF, Zhang SL, Zhu LY, et al. Enhancement of antibacterial activity of tilmicosin against Staphylococcus aureus by solid lipid nanoparticles in vitro and in vivo. Vet J. 2012;191(1):115-120.

20. Misra R, Acharya S, Dilnawaz F, Sahoo SK. Sustained antibacterial activity of doxycycline-loaded poly(D,L-lactide-co-glycolide) and poly(epsilon-caprolactone) nanoparticles. Nanomedicine. 2009;4(5): 519-530.

21. Morishita Y, Yoshioka Y, Takimura Y, et al. Distribution of silver nanoparticles to breast milk and their biological effects on breast-fed offspring mice. ACS Nano. 2016;10(9):8180-8191.
International Journal of Nanomedicine

\section{Publish your work in this journal}

The International Journal of Nanomedicine is an international, peerreviewed journal focusing on the application of nanotechnology in diagnostics, therapeutics, and drug delivery systems throughout the biomedical field. This journal is indexed on PubMed Central, MedLine, CAS, SciSearch $®$, Current Contents $\AA /$ Clinical Medicine,

\section{Dovepress}

Journal Citation Reports/Science Edition, EMBase, Scopus and the Elsevier Bibliographic databases. The manuscript management system is completely online and includes a very quick and fair peer-review system, which is all easy to use. Visit http://www.dovepress.com/ testimonials.php to read real quotes from published authors. 